\title{
Per-operative retinoscopy as a predictor of final post- operative refraction
}

\begin{abstract}
Purpose To assess the accuracy of streak retinoscopy performed at the end of cataract surgery as a predictor of final post-operative error.

Method Retinoscopy was performed on 68 patients as they lay on the operating table after routine cataract extraction and intraocular lens implantation. In each case the predicted postoperative refraction by biometry and the retinoscopy at the end of the operation were compared with the 6 week post-operative subjective refraction.

Results The retinoscopy had a mean difference of $0.6 \mathrm{D}$ (standard deviation of $0.5 \mathrm{D}$ ). The post-operative refraction predicted by biometric measurements had a mean difference of 1.6 D (standard deviation 0.6 D). When corrected for systematic error, $8 \%$ of patients were found to have an error of greater than $2 \mathrm{D}$ as predicted by pre-operative biometry. Prediction by retinoscopy made no error greater than $2 \mathrm{D}$. The accuracy in the retinoscopic prediction of post-operative refraction was significantly better than the biometry using the F-test ( $p=0.001)$. Conclusion Retinoscopy at the end of cataract surgery may be a valuable tool to alert the surgeon to an unexpected refractive error. This would enable immediate intraocular lens exchange, if required.
\end{abstract}

Key words Biometry, Cataract extraction, Emmetropia, Phacoemulsification, Retinoscopy

Patients undergoing cataract surgery now have great expectations, demanding a rapid recovery and good vision without spectacles. ${ }^{1}$ One of the greatest problems facing the cataract surgeon today is the ability to predict post-operative refractive error accurately. Emmetropia is usually desired so that only reading glasses are required. With the development of phacoemulsification and small-incision cataract surgery, many of the traditional problems, particularly the post-operative astigmatism accompanying large sutured wounds that slowed down visual rehabilitation, have been overcome. Accurate biometry (keratometry and A-scan ultrasound) and the correct formula to calculate the power of implant required to obtain the desired post-operative refraction, are essential. Biometric measurement enables good prediction of post-operative refraction in the majority of cases. However, up to $8 \%$ of patients are more than $2 \mathrm{D}$ from the planned refraction. ${ }^{2}$ There are more than 105000 cataract extraction/ lens implantation operations performed within the National Health Service each year. ${ }^{3}$ Thus, there could be as many as 8000 patients each year with unintentional refractive errors of more than $2 \mathrm{D}$.

We postulated that retinoscopy, performed at the end of routine phacoemulsification and lens implantation, could be used as a predictor of post-operative refraction. If this technique could be used to alert the surgeon to an unplanned refractive error, the intraocular lens (IOL) could be changed immediately so that the desired post-operative refraction could be obtained.

\section{Subjects and methods}

Sixty-five consecutive patients, undergoing routine cataract extraction by phacoemulsification by two surgeons (V.F., M.T.), were studied prospectively. Those undergoing combined phaco-trabeculectomy or large-incision extracapsular cataract extraction, and those who had undergone previous trabeculectomy, were excluded from the study.

Manual keratometry was performed using a Haag-Streit keratometer; readings were taken in the two principal meridians. The axial length was measured with an Allergan Humphry 820 A-scanner, using a $10 \mathrm{MHz}$ transducer mounted on a slit lamp. Intraocular lens power was predicted using the SRK2 formula and the Aconstant recommended by the implant manufacturer. The biometry for each patient was reviewed pre-operatively and the appropriate IOL selected; most lenses were chosen to give a post-operative refraction close to emmetropia.
M.J. Tappin

V.M.G. Ferguson

Charing Cross Hospital London, UK

Mr M.J. Tappin Moorfields Eye Hospital City Road London EC1V 2PD, UK Tel: +44 (0)1712533411

Received: 30 August 1998 Accepted in revised form: 31 March 1999 
All patients underwent surgery under local anaesthesia (peribulbar injection) and the surgical method was the same in all cases. A $3.2 \mathrm{~mm}$ two-stage temporal corneal incision was made: the first incision with a diamond knife to approximately two-thirds of the corneal thickness, and the second with a $3.2 \mathrm{~mm}$ keratome, passed horizontally for $1 \mathrm{~mm}$ in the corneal stroma, before entering the anterior chamber. The anterior chamber was then filled with viscoelastic (Healon, Pharmacia) and a paracentesis incision was made so a second instrument could be used during phacoemulsification. Continuous curvilinear capsulorrhexis and hydrodissection were performed. The lens nucleus was removed by the 'divide and conquer' technique. After removal of the lens cortex, a foldable silicone IOL was inserted into the bag through an extended wound of about $3.5 \mathrm{~mm}$. All lenses were Allergan (AMO SI30NB) three-piece foldable lenses with an A-constant of 117.4. The viscoelastic was aspirated and the eye inflated, using balanced salt solution in a 2.5 $\mathrm{ml}$ syringe via a 25 -gauge cannula. The cannula was placed in the paracentesis incision; saline was injected to deepen the anterior chamber until no further backward movement of the lens was observed. The intraocular pressure was assessed by pressing on the globe. If the eye was too hard, a small amount of saline was released from the anterior chamber, whilst care was taken not to allow the IOL to move anteriorly. A subconjunctival injection of antibiotic and steroid was given at the end of the operation.

One surgeon (M.T.), who remained 'masked' to the desired post-operative refraction, performed the retinoscopy immediately after the operation while the patient lay on the operating table. It was performed with a streak retinoscope at $50 \mathrm{~cm}$ from the eye. The surgeon had to approximate his position to that of the visual axis, because all surgery was performed under peribulbar anaesthesia, so fixation by the patient was not possible. The assistant dropped saline on the cornea to prevent drying and to maintain a good retinoscopy reflex.

The patients were reviewed at 1 and 6 weeks postoperatively. At the 6 week visit the departmental optometrist performed a full (objective and subjective) refraction. For comparison with refraction predicted by the biometry results and retinoscopy performed at the end of the operation, the final refraction was converted into the spherical equivalent (cylinder + half the sphere). No patient had a cylinder greater than $2.5 \mathrm{D}$.

\section{Statistical analysis}

The refractions predicted by both biometry and retinoscopy were compared with the actual refraction at 6 weeks. The accuracy of each method of prediction was quantified by calculating the level of error in predicted refraction. The error was determined by the following formulae:

Biometric error $=$ Observed refraction at 6 weeks Expected biometric refraction
Retinoscopic error $=$ Observed refraction at 6 weeks Expected retinoscopic refraction

Comparison of the two sets of errors of prediction for the final post-operative refraction was made using the F-test. The confidence limits were found using tables for the two-sided $1 \%$ level.

\section{Optimisation of lens prediction}

Any method of predicting post-operative refraction may suffer from systematic errors. To study the two methods under optimal circumstances, any systematic error in either method was corrected. In the biometric prediction, the error was corrected by altering the A-constant in retrospect, by leaving the mean error zero, as previously reported. ${ }^{4}$ For retinoscopy, the mean error was subtracted from each value, thus achieving a mean error of zero.

\section{Results}

Sixty-eight patients were included in the study, but the results from 7 patients were excluded because the retinoscopy reflex was difficult to interpret. This was due to a hazy cornea (3 cases) and scissoring of the reflex (4 cases). One case was excluded because the wound was not self-sealing, requiring a single $10 / 0$ nylon suture. A further 8 patients did not attend the optometrist. These patients declined a formal refraction despite three separate invitations by the departmental optometrist. When questioned by telephone they were satisfied with their uncorrected vision and felt refraction unnecessary.

The data from the remaining 52 patients $(81 \%)$ were analysed. The frequency distribution of post-operative refraction at 6 weeks ranged from $-2.25 \mathrm{D}$ to $+3.37 \mathrm{D}$ with a mean refraction of $+0.55 \mathrm{D}(\mathrm{SD}, \pm 1.4 \mathrm{D})$ (Fig. 1). The refraction predicted by biometry ranged from to $-3.89 \mathrm{D}$ to $+1.63 \mathrm{D}$ with a mean of $-1.13 \mathrm{D}(\mathrm{SD}, \pm$ $1.14 \mathrm{D})$ (Fig. 1). The refraction results predicted by retinoscopy ranged from -3.00 to $+3.00 \mathrm{D}$ with a mean value of zero (SD, $\pm 1.5 \mathrm{D}$ ) (Fig. 1). Biometric prediction had a mean error of $+1.69 \mathrm{D}$, and a range from -0.42 to +4.78 D (Fig. 2). Retinoscopic prediction had a mean error of $+0.55 \mathrm{D}$, and a range from -1.0 to $+2.25 \mathrm{D}$ (Fig. 2). When the two methods were optimised,

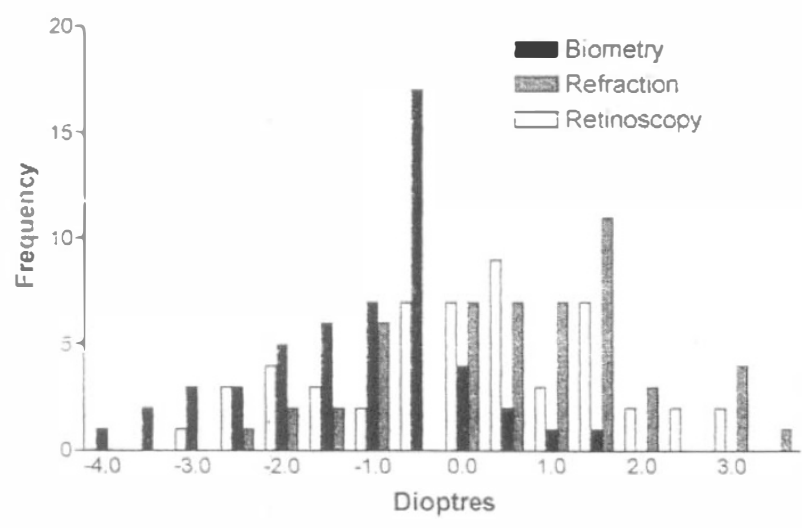

Fig. 1. Retinoscopy, refraction and biometry data. 


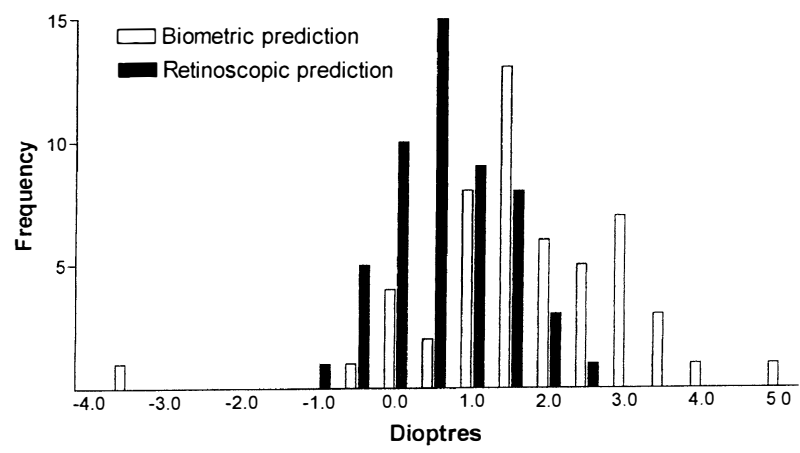

Fig. 2. Error in prediction of post-operative refraction.

removing any systematic error from either technique, the biometric error range was -5.11 to $+3.09 \mathrm{D}(\mathrm{SD}, \pm 1.33$ D) (Fig. 3). The corrected retinoscopy error range was -1.55 to $+1.7 \mathrm{D}$ (SD, $\pm 0.7 \mathrm{D}$ ) (Fig. 3).

\section{Discussion}

Following uncomplicated cataract surgery we found that the error in desired refraction at 6 weeks was from -3.42 to $+4.78 \mathrm{D}$, with a mean of $+1.6 \mathrm{D}$ (SD, 1.3). This represents a similar range to other studies, which have found a spread of -4.09 to +2.55 D. ${ }^{5}$

We compared the refraction anticipated by biometry with the actual refraction and found a hypermetropic shift. After optimising the biometric predictions, error ranged from -5.1 to $+3.1 \mathrm{D}$.

The second method of predicting post-operative refraction was by retinoscopy; the range of error was -1.55 to $+1.7 \mathrm{D}$. After correcting both methods for systematic error, retinoscopy was significantly $(p<0.001)$ better at predicting the post-operative refraction than biometry. There was a greater spread of error in the biometry than from retinoscopy. Eight per cent of patients had a final refraction greater than $2 \mathrm{D}$ from that predicted by biometry, and $32 \%$ greater than $1 \mathrm{D}$. In comparison, retinoscopy made no errors greater than 2 $\mathrm{D}$, and only $17 \%$ had predicted errors of more than $1 \mathrm{D}$ from the final subjective refraction.

Retinoscopy is not always possible at the end of cataract surgery. We found that it was not possible to perform retinoscopy on 7 patients ( 4 hazy cornea, 3 scissoring reflex).

There are several sources of error in per-operative retinoscopy. Systematic errors may be due, firstly, to overestimation of the distance from the retinoscope to the patient, secondly, to over-deepening of the anterior chamber, and thirdly, to changes in shape of the posterior pole due to peribulbar anaesthesia.

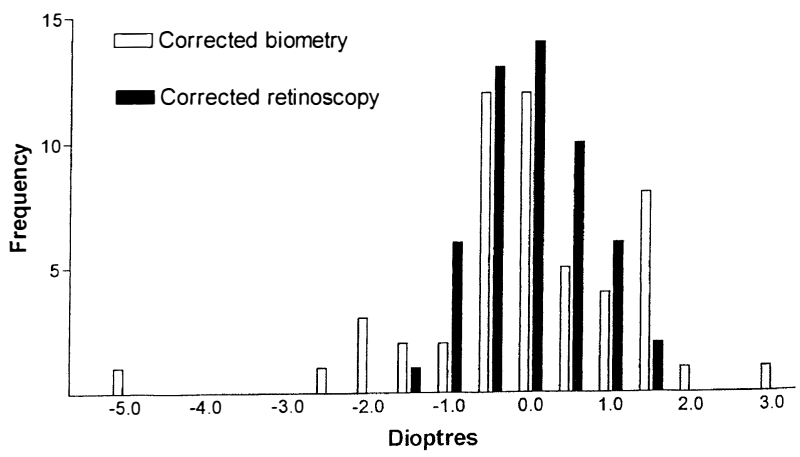

Fig. 3. Corrected errors in predicted refraction.

Non-systematic errors may be due, firstly, to an inconsistent anterior chamber depth at the end of the operation. Secondly, as all surgery was performed under peribulbar anaesthesia, the patients were unable to fixate and the visual axis had to be estimated by the surgeon performing retinoscopy. Thirdly, the dilated pupil with the relatively small diameter optic of foldable IOLs may lead to retinoscopic errors.

We decided to deepen the anterior chamber as described, in an attempt to provide a consistent anterior chamber depth, with an easily observed end-point. This may reduce variations in anterior chamber depth in some cases, with a hypermetropic shift.

Surgical and retinoscopic techniques vary, so it is important for the individual surgeon to assess his or her own accuracy in predicting post-operative refraction before using this method.

This study demonstrates that those patients with errors in their post-operative refraction greater than $2 \mathrm{D}$ may be picked up using retinoscopy at the end of cataract surgery. We suggest that this may be useful for patients who have inconsistent biometry, or very long or short eyes leading to less accurate biometric prediction of IOL power. The surgeon could consider early IOL exchange in order to reduce the number of surprise postoperative refractive results.

\section{References}

1. Nordan LT, Ernest P, Fine H, et al. Letter to the editor. Ophthalmology 1991;98:1319-20.

2. Hovding G, Natvik $C$, Sletteberg $O$. The refractive error after implantation of a posterior chamber intraocular lens. Acta Ophthalmol 1994;72:612-6.

3. Desai P. The National Cataract Surgery Survey. 2. Clinical outcomes. Eye 1993;7:489-94.

4. Olsen T, Thim K, Corydon L. Accuracy of the newer generation intraocular lens power calculation formulas in long and short eyes. J Cataract Refract Surg 1991;17:187-93.

5. Olsen T, Bargum R. Outcome monitoring in cataract surgery. Acta Ophthalmol Scand 1995;73:433-75. 